\title{
Cuatro destinos del guerrero: teorías de la guerra indígena en las tierras bajas sudamericanas
}

\section{Diego Villar}

\section{(2) OpenEdition \\ 1 Journals}

\section{Electronic version}

URL: http://journals.openedition.org/corpusarchivos/1395

DOI: 10.4000/corpusarchivos. 1395

ISSN: 1853-8037

\section{Publisher}

Diego Escolar

\section{Electronic reference}

Diego Villar, «Cuatro destinos del guerrero: teorías de la guerra indígena en las tierras bajas sudamericanas », Corpus [En línea], Vol 5, No 1 | 2015, Publicado el 30 junio 2015, consultado el 07 mayo 2020. URL : http://journals.openedition.org/corpusarchivos/1395; DOI : https://doi.org/ 10.4000/corpusarchivos.1395

This text was automatically generated on 7 May 2020. 


\title{
Cuatro destinos del guerrero: teorías de la guerra indígena en las tierras bajas sudamericanas ${ }^{1}$
}

\author{
Diego Villar
}

1 En un artículo publicado en La Prensa, en plena guerra del Chaco, Alfred Métraux se pregunta si hay alguna continuidad entre el conflicto entre Bolivia y Paraguay y la guerra tradicional de los indígenas del Pilcomayo (Métraux 1933, cf. Córdoba 2013). No pretendo resolver aquí tamaña cuestión ni menos aún revivir la discusión bizantina sobre qué es lo que constituye o no la guerra (Fried et al.1968, Otterbein 1999, Gat 1999, Otto et al. 2006). Por el momento, una definición agustiniana -si nadie me pregunta qué es la guerra lo sé, si me lo preguntan no lo sé- deberá bastar para identificar pragmáticamente, a grandes rasgos, cuatro tipos de explicación propuestos por la antropología a la hora de entender la guerra indígena en las tierras bajas sudamericanas. Lejos de las ambiciones analíticas de los modelos predictivos se trata de exponer cuatro grillas de interpretación de lo bélico como si fueran testimonios nativos, evaluando su productividad a la hora de iluminar los estudios de caso: i.e. cómo definen, escogen y disponen los datos, cómo establecen la relevancia de las asociaciones, cómo recortan los parámetros pertinentes de abstracción y de comparación. Sin embargo, antes de comenzar, conviene aclarar algunos puntos: primero, analizo la literatura etnográfica pero no necesariamente la arqueológica; segundo, no propongo un análisis exhaustivo de las teorizaciones en cuestión sino apenas la posibilidad de identificar líneas de fuerza que vertebran su explicación de la guerra amerindia; tercero, cuando hablo de "tierras bajas" me refiero especialmente a las áreas culturales del Chaco y de la Amazonía; por último, la idea misma de "guerra indígena" me remite -aunque sea en principio- a los enfrentamientos armados entre distintos grupos o subgrupos indígenas, y no necesariamente entre estos y diversos actores colonizadores coloniales, republicanos, privados, etc. 


\section{La guerra contra el Estado}

2 Célebre por sus reverberaciones filosóficas, una primera teorización de la guerra amerindia es la propuesta por Pierre Clastres. Como casi todo en la moderna etnología francesa, su interpretación parte de la obra de Lévi-Strauss. En efecto, en un artículo titulado "Guerra y comercio entre los indios de América del Sur", Lévi-Strauss (1943) había adelantado uno de sus argumentos fundamentales: la guerra y el comercio son dos aspectos indisociables de un mismo problema, el intercambio. Los intercambios económicos son guerras potenciales, resueltas pacíficamente; las guerras son transacciones comerciales fallidas. En esta variación estructuralista de la fórmula clausewitziana -la guerra como continuación del intercambio por otros medios-, lo bélico se presenta como mecanismo de resolución de una crisis que interrumpe la paz, la cual se presenta como el estado primario, inicial o básico de la sociabilidad. Es aquí donde Clastres hinca el diente: si la guerra no es más que un caso particular de un sistema general de relaciones que constituye la sociedad (el intercambio), no hay una autonomía real para la esfera de la violencia; o, en otras palabras, si la guerra es todo, termina siendo nada. La teoría de Lévi-Strauss define la guerra en términos negativos, sin concederle una especificidad propia. En cambio Clastres insiste en la necesidad de definir la guerra en positivo, como una "estructura de la sociedad primitiva" que es "omnipresente" y no puede reducirse a un intercambio abortado (Clastres 1987, pp. 181-216).

3 ¿Dónde encuentra Clastres la positividad de lo bélico? De forma paradójica, en una nueva negatividad. 0 más bien en una suerte de dispositivo de obstrucción sociológica. Hay que pensar, aquí, en el típico cisma que reporta la etnografía amerindia: cuando una sociedad sobrepasa cierto nivel demográfico lo más probable es que sus miembros peleen entre sí y el grupo se divida. La guerra, dice Clastres, "es el principal medio utilizado por la sociedad primitiva para impedir el cambio social" (1987, p. 212). Como en el caso legendario del mesianismo tupí-guaraní, se trata de un mecanismo centrífugo que opera como un antídoto endógeno, un anticuerpo que obtura la fuerza centralizadora de lo social y bloquea el surgimiento amenazante de "lo Uno" (el Estado). Clastres desempolva en este punto la tesis duméziliana de las tres funciones de la ideología indoeuropea, encarnada en la tríada Júpiter-Marte-Quirino (soberanía, guerra, producción); aunque, si Dumézil analizaba la función guerrera en los mecanismos de representación de sociedades que procuran mantenerse divididas, él pretende en cambio comprender cómo actúa esa misma función en las sociedades que se pretenden igualitarias (Clastres 1987, p. 218) ${ }^{2}$.

4 En páginas memorables, Clastres relee el siglo de oro de la etnografía jesuita en el Chaco. Las obras de Paucke, Lozano, Dobbrizhoffer o Sánchez Labrador ponen en escena "la desgracia del guerrero salvaje" describiendovívidamentela voracidad bélica, el código de honor o la vocación de prestigio de las "cofradías" o "castas" guerreras de abipones, mocovíes y caduveos. La guerra chaqueña es una carrera abierta al talento. Cada hazaña lleva a la otra, el prestigio es puesto en juego en cada enfrentamiento y la gloria jamás es suficiente: hay una "insatisfacción permanente" del guerrero "condenado al activismo" y a "huir para adelante" en un vértigo individualista, desbocado, insensato, que lo hace ir a la batalla solo contra todos -como el cacique toba Kalali'in, que se infiltra por las noches en los campamentos nivaclés para degollar un par de enemigos hasta que finalmente lo capturan y muere torturado, sin emitir 
sonido. La lógica de la explicación de la guerra, así, es más o menos la misma que la del mesianismo tupí-guaraní: "la sociedad" impide que el guerrero acapare poder y se haga "señor". Ciertamente le concede un efímero prestigio, pero a la vez la carrera bélica no deja de ser una empresa disfuncional, suicida, un "ser-para-la-muerte" que jamás logra afianzar un aparato centralizado de poder (Clastres 1987, p. 217-256).

El potencial heurístico de la antropología política clastreana es tan conocido como las denuncias de sus críticos, no menos violentos que los guerreros chaqueños: para unos la teoría de los anticuerpos "contra el Estado" esboza una visión de la acción social hiperculturalista; para otros compone una épica anti-evolucionista de ribetes utópicos; para otros, por fin, no es más que un enunciado metafísico. Más allá de las idiosincrasias, el punto que conviene retener es que la guerra amerindia se explica en función de una lógica interna, endógena, inmanente, autogenerada, por momentos casi trascendental, que caracteriza a toda "sociedad primitiva" (Clastres 1987, 2009)3.

\section{La guerra constituyente}

6 No se trata, sin embargo, de la única caracterización de la guerra indígena en términos culturalistas. Un segundo tipo de lectura, también de obvia inspiración lévi-straussiana, sugiere que todas las formas de enfrentamiento bélico (la caza de scalps chaqueña, el exocanibalismo chiriguano o juruna, el endo canibalismo aché, cashinahua o yanomami, la caza de cabezas mundurucú o jíbaro) comparten una misma lógica profunda, cifrada en una serie de postulados más o menos recurrentes.

En primer lugar la guerra supone la presencia de adversarios ni tan cercanos ni tan lejanos, ni tan iguales ni tan diferentes; así, por ejemplo, los shuar no pelean con sus parientes ni con los cocamas, los canelos o los blancos, sino más bien con los demás representantes de lo que podríamos llamar el "macroconjunto jíbaro": los achuar, los candoshi, los aguaruna, etc. La guerra implica una afinidad electiva con ciertos enemigos preferenciales que comparten un determinado horizonte de inteligibilidad: no necesariamente la lengua, la cultura o las costumbres consideradas como un todo, pero sí el idioma simbólico del canibalismo, la vendetta, la caza de cabezas o de scalps. En segundo lugar los propios actores explican la guerra apelando a ideas más o menos explícitas sobre la captura, la absorción o la asimilación de fuerzas/poderes del enemigo. En tercer lugar la teoría nativa encubre una explicación más profunda, subyacente o implícita, que apela una vez más a la lógica del intercambio aunque no pasa tanto por la reproducción de la "sociedad", a la manera funcionalista, sino más bien por la necesidad de reproducir el cosmos todo: la identidad, la memoria propia y ajena, el cuerpo físico y social, la fertilidad, los ciclos naturales y sociales, etc. En cierta forma, pues, la guerra no es algo que "sucede" sino que constituye un sistema autosustentable, estructuralmente necesario para que la sociedad y el cosmos puedan reproducirse ${ }^{4}$.

8 Las inflexiones argumentales de esta grilla de lectura son perceptibles en un caso paradigmático: los tupinambás de la costa brasileña. Por medio de crónicas como las de Hans Staden, Anchieta, Jean de Léry, André Thévet o Claude d'Abbeville, de los grabados de Teodoro de Bry o de los clásicos ensayos de Montaigne, estos indígenas componen el arquetipo del salvajismo para el imaginario colonial europeo de los siglos 16 y 17. Hay imaginerías recurrentes: por un lado la guerra intestina, cíclica, incesante; por otra parte el drama escalofriante del sacrificio de los cautivos y la antropofagia 
ritual. No hace falta reiterar demasiado la famosa secuencia de episodios que componen "la tragedia caníbal". Un grupo tupinambá captura a un guerrero, lo lleva cautivo a la aldea, le da una esposa, lo domestica. El cautiverio no es una deshonra; por el contrario, el prisionero acepta orgullosamente el destino del guerrero y jamás se fuga. Finalmente, en una gran celebración colectiva, el prisionero vuelve a ser enemigo: es perseguido, apresado, insultado mientras anuncia altivamente a sus captores que lo vengarán sus parientes y que los matadores de hoy son las víctimas de mañana. Las víctimas de mañana lo ejecutan de un macanazo en la nuca. El matador suma un nuevo nombre, gana prestigio y guarda ayuno mientras sus parientes desmiembran, cocinan y comen la víctima (Combès 1991).

9 Las explicaciones clásicas del rito son las del etnólogo suizo Alfred Métraux (1928) y el sociólogo brasileño FlorestanFernandes (2006). El ciclo de las venganzas caníbales es un rito sacrificial que forma parte del "culto a los ancestros", reparación simbólica que devuelve al grupo la "sustancia vital" que los enemigos le habían quitado en una matanza anterior. El ritual permite al grupo salir de la "dependencia" o "heteronomía mística" y restaurar el equilibrio del "nosotros colectivo" (Bossert y Villar 2007,Bossert 2012). Para la moderna etnología francobrasileña, en cambio, la antropofagia tupinambá es mucho más que eso; se trata del tipo paradigmático de relación social -la llamada "predación"- que presuntamente rige las tierras bajas sudamericanas, y muchas veces más allá. Partiendo del dogma lévi-straussiano de la ouverture à l'autre se invierte entonces el planteo clásico. Más que una vendetta (i.e. un fenómeno sociológico o jurídico), la guerra tupinambá es una predación ontológica (i.e. algo cosmológico, ontológico, metafísico). Los grupos sociales no preexisten a la guerra sino que se constituyen como tales, justamente, a través de ella: necesito al otro para poder reproducirme puesto que la relación con el enemigo -o la alteridad en sentido laxoprecede, constituye, posibilita en todos los casos mi propia identidad. Lo que asimilo no es ya la fuerza, la energía, la vitalidad o el poder de mi enemigo sino, justamente, su posición misma como enemigo; es decir, su propio punto de vista sobre mí. Al fin y al cabo mi identidad no es más que la suma de perspectivas que tienen los otros (extranjeros, enemigos, animales, espíritus, muertos) sobre mí mismo. En esta clave analítica, pues, se comprende por qué el canibalismo no solo constituye la forma paradigmática de la relación predatoria sino, incluso, de la relación misma (Carneiro da Cunha y Viveiros de Castro 1985,Combès 1991,Viveiros de Castro 1992, Fausto 2007).

\section{La materialidad de la guerra}

Un tercer tipo de explicación de la guerra amerindia plantea preguntas distintas. ¿Qué pasa cuando esa maquinaria bélica autosustentable se desarticula por razones demográficas, por factores de cambio social como la colonización, por cualquier forma de -digamos así- entrar en la historia? ¿Existió acaso esa maquinaria, o es más bien la construcción de ciertos profesores con imaginación entusiasta? ¿Cómo explicar la experiencia bélica de las sociedades indígenas chaqueñas, desarticuladas dramáticamente por la guerra del Chaco entre Bolivia y Paraguay, que se sirven no obstante del conflicto para realimentar antiguas enemistades? (Richard 2008, Capdevilaet al. 2010). ¿Cómo entender el uso colonial de indígenas en tropas de "indios amigos" (los guaraníes en las expediciones de Alejo García, Irala, Cabeza de Vaca o Ñuflo de Chaves durante el siglo 16), en cuadros organizados en las milicias misionales 
o incluso como mercenarios (los mundurucú de la Amazonía)? (Susnik 1971,Susnik y Chase-Sardi 1995,Ferguson 1990, Carvalho 2002, Martínez 2012). ¿Peleaban entre ellos antes de la llegada de los españoles? Y si lo hacían, ¿peleaban igual? ¿Qué pasa con los ciclos de vendettas de los jíbaros lato sensu cuando los misioneros jesuitas y salesianos introducen las armas de fuego? (Steel 1999). ¿O con los indómitos chiriguanos del piedemonte andino, que pasan de devorar a los siervos chanés en el rito antropofágico a venderlos como esclavos? (Díaz de Guzmán 1979 [1617-1618], p. 78). ¿O bien, al otro extremo del continente, con los mismos tupinambás, protagonistas por antonomasia de las elucubraciones neoestructuralistas? Porque ciertamente es posible hacer otra lectura de las fuentes del siglo de oro tupí a la luz de las demandas del mercado esclavista que abastece las plantaciones de caña de azúcar o de la propia guerra colonial entre Francia y Portugal (Ferguson 1990, p. 241). O sea que, en sus variantes más moderadas, esta grilla de lectura alternativa no solo nos propone ambigüedades y zonas grises en la tesis de una guerra autorregulada, sino que en sus versiones más extremas llega a afirmar sin ambages que la guerra indígena no es más que el epifenómeno de dichas disrupciones externas. La guerra como coyuntura pura.

11 Un buen ejemplo etnográfico es el de los caduveos, presentados tempranamente por el propio Lévi-Strauss (1958) como caso ejemplar del análisis estructural. Pero lo cierto es que ese mismo caso permite matizar aquella afirmación de Clastres: que la guerra indígena es un medio de impedir el cambio social. Desde los informes del jesuita Sánchez Labrador a los reportes etnológicos de Branislava Susnik, estos indígenas son famosos por la adopción del llamado "complejo ecuestre" y también por sus llamativos mecanismos de estratificación social. En efecto, la caduveaes una sociedad "aristocrática" con una tripartición duméziliana o hasta medieval: primero los nobles, jerarquizados a su vez en nobles hereditarios y otros ennoblecidos por matrimonios hipergámicos; segundo los guerreros, con sus prerrogativas especiales, sus cofradías y sus rituales de iniciación, que permiten al ascenso social; finalmente los siervos, jerarquizados a su vez, que provienen de diferentes parcialidades vecinas (terenas, guanás, guaraníes, chamacocas, caingangues). El propio Lévi-Strauss reporta ejemplos elocuentes de la obsesión caduvea con la estirpe, la raigambre y la alcurnia: las mujeres blancas capturadas no tienen nada que temer de los indígenas porque ningún caduveo mancillaría su sangre poniéndoles un dedo encima; las damas rehúsan visitar a la esposa del virrey porque solo la reina de Portugal sería digna de ellas; o la mujer que declina la invitación del gobernador de Mato Grosso para ir a Cuiabá porque supone que el funcionario quiere casarse con ella y no quiere ofenderlo con su rechazo. La ideología jerárquica cuenta incluso con una legitimación mítica. Cuando el ser supremo Gonoenhodí crea a los hombres saca de una tinaja a los guanás y les da agricultura, luego saca a las otras tribus y les regala la caza, y al hacerlo olvida a los caduveos en el fondo del recipiente: como nada queda para ellos les concede el derecho de oprimir y explotar a los demás. En la praxis esta ideología de rango se manifiesta en una curiosa aversión por lo emocional, por lo sentimental, casi por la misma biología. Los caduveos dicen sentir "asco" por la procreación y no son raros entre ellos el aborto y el infanticidio: la perpetuación del grupo ocurre de hecho por captura y por adopción más que por generación -así, el propio Lévi-Strauss calcula que, a principios del siglo 19, apenas el 10\% del grupo es caduveo "de sangre" (1958, p. 155-212). Y sin embargo, más allá de la adopción del caballo y de las implicancias sociopolíticas del horse complex, las fuentes sobre el Chaco y el Mato Grosso muestran una y otra vez que los caduveos no solo apresan esclavos para reproducir su propio sistema social: alternan los papeles de 
aliados o enemigos de españoles y portugueses en tiempos coloniales y luego de los paraguayos o brasileños en tiempos republicanos, siempre de acuerdo con los intereses del momento, tomando esclavos para sí pero a la vez para comerciar con el frente colonizador. $\mathrm{O}$ sea que, al menos en este caso, la guerra indígena parece ligarse con el motor de la diferenciación social de un modo bastante más complejo que el que imaginaba Clastres ${ }^{5}$.

12 Si las explicaciones clastreanas o neoestructuralistas llevan la explicación de la guerra a un extremo analítico (reducir lo bélico a una causa, función o sentido internos), este nuevo tipo de interpretaciones suele conducir al otro: desplaza por completo la agencia bélica hacia el exterior del mundo indígena. En esta clave hermenéutica la guerra no es ya una institución autorregulada, endógena, en definitiva propia, sino mera coyuntura provocada por el interés de mantener o mejorar determinadas condiciones materiales de existencia en condiciones históricamente cambiantes, y especialmente en escenarios de contacto - un ejemplo claro de esta grilla de lectura es el de Brian Ferguson (2001, p. 100-104), que analiza la guerra yanomami como consecuencia directa de la intervención de misioneros, militares, antropólogos y ONG. Y el caso yanomami, justamente, nos lleva a un último tipo de explicación de lo bélico.

\section{Guerra biológica}

La interpretación biológico-evolutiva de la guerra tal vez sea menos conocida para nosotros pero tiene sentido en el marco institucional del campo antropológico norteamericano, dividido tradicionalmente en arqueología, antropología lingüística, antropología cultural y antropología biológica. Justamente hablando de guerra hay ahí una suerte de conflicto endémico entre la antropología biológica y la cultural. Si los enfoques culturales basan sus hallazgos en tendencias inferidas a partir de conjeturas, intuiciones o generalizaciones que se asumen como diagnósticas, la antropología evolutiva o biológica enfatiza más bien los hard facts (p. ej. estadísticas); por citar un solo ejemplo, se calcula meticulosamente la tasa estadística de muertes bélicas sobre muertes totales en las "sociedades simples", que al parecer ronda el 15\% (y el 25\% para los varones) (Gat 1999, p. 595).

14 El conflicto de las interpretaciones estalla en la "controversia yanomami", que a esta altura es ya una disciplina en sí misma y abarca centenares de libros, artículos y sitios de internet. La enorme mayoría de las referencias gira en torno de la figura de Napoleón Chagnon -según el New York Times, "nuestro antropólogo más controvertido". Chagnon es un antropólogo biológico polémico, con un perfil altísimo, políticamente incorrecto, que se regodea posando con escopeta y sombrero de cowboy al estilo Indiana Jones y vende miles de libros diciendo cosas como que "los mayores obstáculos para la teoría de la evolución son las religiones organizadas y la antropología cultural" (Eakin 2013, p. 32). Como era de esperar los antropólogos culturales contraatacan acusándolo de forzar o fabricar los datos, de clamorosas faltas de ética (p. ej. obligar a los indígenas a revelar sus nombres secretos o pagarles a terceros para que revelen los nombres de los demás), o bien de "esencializar" o "exotizar" a los yanomamis como una sociedad de la violencia.

15 Un punto álgido en tanta discordia es un artículo publicado por Chagnon (1988) en Science, en el que afirma que los yanomamis viven en un estado de guerra crónica. Analizando casi trescientas historias de vida de unokai (aquel que ha matado a otra 
persona y cumple el tradicional ritual purificatorio para espantar el espíritu de la víctima), Chagnon concluye que estos tienen más esposas e hijos que los hombres normales en proporción de tres a uno. Por lo tanto, a más guerra, más éxito reproductivo. La guerra no es pues una adaptación más entre otras: "La violencia es una fuerza potente en la sociedad humana y bien puede ser la principal fuerza que impulsa la evolución de la cultura" (Chagnon 1988, p. 985).

Las críticas al planteo de Chagnon son de varios órdenes. En primer lugar hay una serie de problemas de traducción: la definición de unokai como "aquel que mató con tal o cual método" encubre en rigor otros valores asociados culturalmente con el coraje, con la suerte en la caza, con la generosidad y, en cuanto a la capacidad concreta para la violencia, con la capacidad de dañar mediante la hechicería. En segundo lugar Chagnon omite las estadísticas de padres muertos de niños vivos, cuando el sentido común indicaría que quienes no mueren prematuramente en la guerra tienen más chances de llegar a viejos y de procrear que aquellos que mueren jóvenes. En tercer lugar, no distingue la "unokai-dad" de la "normalidad" yanomami por grupos de edad, y entonces no queda claro si los guerreros tienen más hijos por ser unokai o simplemente por ser adultos y viejos, o si tienen más esposas e hijos por ser unokai o simplemente por ser caciques (todos los jefes son unokai pero no todos los unokai son necesariamente jefes y, como en casi todas las tierras bajas sudamericanas, la poliginia yanomami es prerrogativa cacical). En cuarto lugar, Chagnon no distingue con claridad al pater del genitor, i.e. la paternidad social de la biológica: así, los grandes guerreros tienen muchas esposas que a la vez tienen amantes jóvenes, que suelen darles hijos, a los que se clasifica como progenie de unokai -lo cual socialmente no supondría ningún problema pero sí resulta incómodo para un argumento que liga causalmente el prestigio guerrero con la reproducción biológica (Albert 1989, 1990; Ferguson 2001, pp. 106-101). Más allá del perfil controversial de Chagnon, pues, las últimas décadas han visto acumularse una serie considerable de críticas en función de la consistencia interna de su modelo ${ }^{6}$.

\section{Una ciencia de la guerra}

17 En las tierras bajas sudamericanas el destino del guerrero indígena parece limitarse a morir en una busca maníaca del prestigio social, a morir para perpetuar al otro y en el ínterin a sí mismo, a morir por circunstancias externas que no siempre termina de entender o bien a morir para obtener más esposas e hijos. Más allá de si la muerte es autogenerada o impuesta podemos preguntarnos qué presupuestos antropológicos anidan tras cada una de estas cuatro grillas exegéticas. Pues si las dos primeras revelan aspiraciones filosóficas más o menos encubiertas, las dos últimas se apoyan en razones más prosaicas. En efecto, en las teorías materialista y biológica la categoría de "guerra" tiene un sentido más o menos familiar para nosotros; en última instancia, las motivaciones son básicamente las mismas para todos los hombres (consciente o no el guerrero busca mejorar su estatus material, reproductivo, etc.). La segunda interpretación (neoestructuralista), en cambio, pone en escena un actor que necesita combatir por una razón completamente diferente: para constituir su propia identidad personal y colectiva al reciclar la alteridad. Resta, por último, la alternativa más inquietante, la formulación clastreana, que postula un guerrero que mata para preservar su sociedad tal como está, en una suerte de equilibrio que precisamente 
supone a la propia guerra como "estructura básica"; con lo cual, abusando un poco de la transitividad, podríamos decir que mata para seguir matando.

Sea como fuere, a la hora de analizar la guerra indígena este breve repaso nos obliga a pensar en qué medida podemos ir más allá de las declaraciones programáticas y trascender realmente la epistemología teleológica (Hallpike 1973) ${ }^{7}$.

Por otra parte, impone una segunda constatación. No hemos avanzado mucho más allá de lo que aventuraba Métraux en 1933. ¿Con quién se hace la guerra? En general con adversarios preferenciales ni tan cercanos ni tan distantes, ni tan similares ni tan distintos, con los cuales se comparten ciertos códigos. ¿Qué es la guerra? Un hecho social total con facetas prácticas, tecnológicas, logísticas, económicas, simbólicas, productivas, ideológicas, etc. ¿Por qué se hace la guerra? Según los actores el casus belli puede ir desde pleitos jurisdiccionales (p. ej. cotos de caza, pozos de agua, espacios de pesca) hasta la vendetta, del mero afán de conseguir alimentos, armas o herramientas a la acumulación sistemática de prestigio, poder, nombres, cautivos, mujeres o niños. ¿Cómo se hace la guerra? Evitando en lo posible el enfrentamiento abierto, "simétrico" o "directo" en los viejos términos de Basil Lidell-Hart; prefiriendo en todos los casos la emboscada, el raid sorpresivo por la madrugada o cualquier otra estrategia que minimice el riesgo y garantice la mayor probabilidad de éxito ("La táctica de los indios consiste esencialmente en sorprender y no ser sorprendidos", dice Métraux en 1933; cf. Liddell-Hart 1989, Gat 1999). En lo que varía luego cada orientación analítica es en la explicación de la causa profunda, subyacente, determinante, lo que podríamos llamar el locus de una agencia bélica que parece escapar a la conciencia de los actores para afincarse en la estructura cosmológica, en un anticuerpo misterioso contra la centralización política, en evaluaciones grupales de costos y beneficios en tiempos de cambio social o incluso en una serie de adaptaciones evolutivas. En estas condiciones lo más razonable es pues evitar el dogmatismo teórico, moderar el entusiasmo explicativo y tomar las teorías generalistas de la guerra amerindia con todas las salvedades aplicables a cualquier tipo ideal: las formas bélicas particulares pueden clasificarse en varias de las categorías a la vez, y las grillas interpretativas no son necesariamente excluyentes ni más que artificios heurísticos que el observador construye para resaltar contra ellos la particularidad de cada modalidad bélica (Weber 1993). Después de todo, como dicen que decía Liddell-Hart, sin duda hay una ciencia de la guerra: solo nos falta descubrirla.

\section{BIBLIOGRAPHY}

Albert, B. (1989). Yanomami “Violence": Inclusive Fitness or Ethnographer's Representation? Current Anthropology, 30 (5), 637-640.

Albert, B. (1990). On Yanomami Warfare: Rejoinder. Current Anthropology, 31, 558-563. 
Bossert, F. (2012). La antropofagia tupinambá: del sacrificio a la predación. Ponencia, Seminario Repensando el Perspectivismo Amerindio, Instituto de Ciencias Antropológicas, Facultad de Filosofía y Letras, Universidad de Buenos Aires.

Bossert, F. y Villar, D. (2007). La etnología chiriguano de Alfred Métraux. Journal de la Société des Américanistes, 93 (1), 127-166.

Campagno, M. (Ed.) (2014). Pierre Clastres y las sociedades antiguas. Buenos Aires: Miño y Davila. Capdevila, L., Combès, I., Richard, N. y Barbosa, P. (2010). Los hombres transparentes. Indígenas y militares en la Guerra del Chaco (1932-1935). Cochabamba: ILAMIS-Itinerarios (Scripta Autochtona 5). Cardoso de Oliveira, R. (1976). Do Índio ao Bugre: o processo de assimilação dos Terenas. Rio de Janeiro: Museu Nacional do Índio.

Carneiro da Cunha, M. y Viveiros de Castro, E. (1985). Vingança e temporalidade: os Tupinamba. Journal de la Société des Américanistes, 71, 191-208.

Carvalho, S. (2002). Chaco: encruzilhada de povos e 'melting pot' cultural, suas relações com a bacia do Paraná e o sul mato-grossense. En Carneiro da Cunha, M. (ed.), História dos índios no Brasil(pp. 457-474). San Pablo: Companhia das Letras.

Chagnon, N. (1988). Life Histories, Blood Revenge, and Warfare in a Tribal Population. Science (n.s.), 239 (4843), 985-992.

Chaumeil, J-P. (1985). Échange d'énergie: guerre, identité et reproduction sociale chez les Yagua de l'Amazonie péruvienne. Journal de la Société des Américanistes, 71, 143-157.

Clastres, P. (1987). Investigaciones en antropología política. Barcelona: Gedisa.

Clastres, P. (2009). La sociedad contra el Estado. Buenos Aires: Caronte.

Combès, I. (1991). La tragédie cannibale chez les anciens Tupi-Guarani. París: Presses Universitaires de France.

Combès, I. y Villar, D. (2013). La Tierra sin Mal. Leyenda de la creación y destrucción de un mito. Tellus, 24, 201-225.

Cordeu, E. (2004). Los Tomaráxo y los 'indios cavalheiros' (Caduveo): aportes para la historia de un sistema intertribal. En Cipolletti, M. S. (Ed.), Los Mundos de abajo y los mundos de arriba: Individuo y sociedad en las tierras bajas, en los Andes y más allá (pp. 275-312). Quito: AbyaYala.

Córdoba, L. (2013). Alfred Métraux y los tobas del oeste formoseño (Argentina). Ponencia, Alfred Métraux. Relectures transatlantiques. Colloque organisé en hommage à Alfred Métraux (1902-1963), Musée du quai Branly/École des Hautes Études en Sciences Sociales, París.

Deleuze, G. y Guattari, F. (2002). Mil mesetas. Capitalismo y esquizofrenia. Valencia: Pre-Textos.

Descola, P. (1993). Les affinités sélectives. Alliance, guerre et prédation dans l'ensemble jivaro. L'Homme, 33 (126-128), 171-190.

Díaz de Guzmán, R. (1979[1617-1618]). Relación de la entrada a los Chiriguanos. Santa Cruz: Fundación cultural "Ramón Darío Gutiérrez".

Dumézil, G. (1969). Heur et Malheur du guerrier. Aspects mythiques de la fonction guerrière chez les IndoEuropéens. París: Presses Universitaires de France.

Eakin, E. (2013). Who Are the Real Savages? The New York Times, 17 de febrero, Nueva York: 32.

Erikson, P. (1986). Altérité, tatouage et anthropophagie chez les Pano: la belliqueuse quête du soi. Journal de la Société des Américanistes, 72, 185-210. 
Fausto, C. (2007). Feasting on People. Eating Animals and Humans in Amazonia. Current Anthropology, 8 (4), 497-530.

Fernandes, F. (2006). A função social da guerra na sociedade tupinambá. San Pablo: Globo.

Ferguson, B. (1990). Blood of the Leviathan: Western Contact and Warfare in Amazonia. American Ethnologist, 17, 237-257.

Ferguson, B. (2001). Materialist, cultural and biological theories on why Yanomami make war. Anthropological Theory, 1(1), 99-116.

Fried, M., Harris, M. y Murphy, M. (Eds.) (1968).War: The Anthropology of Armed Conflict and Aggression. Nueva York: The Natural History Press.

Gat, A. (1999).The pattern of fighting in simple, small-scale, prestate societies. Journal of Anthropological Research 55, 563-583.

Goés Neves, E. (2009). Warfare in Precolonial Central Amazonia. When Carneiro meets Clastres. En Nielsen, A. y Walker, W. (Eds.), Warfare in Cultural Context. Practice, Agency and the Archeology of Violence (pp. 140-164). Tucson: The University of Arizona Press.

Hallpike, C. R. (1973). Functionalist Interpretations of Primitive Warfare. Man (n.s.), 8 (3), 451-470. Heinen, D. e Illius, B. (1996). The Last Days of El Dorado: A Review Essay on Yanomami Warfare. Anthropos, 91 (4/6), 552-560.

Kersten, L. (1968). Las tribus indígenas del Gran Chaco hasta fines del siglo XVIII. Una contribución a la etnografía histórica de Sudamérica. Resistencia: Universidad Nacional del Nordeste.

Lévi-Strauss, C. (1943). Guerre et commerce chez les Indiens de l'Amérique du Sud. Renaissance, 1 $(1 / 2), 122-139$.

Lévi-Strauss, C. (1958). Tristes trópicos. Buenos Aires: EUDEBA.

Lidell-Hart, B. (1989). Estrategia. La aproximación indirecta. Madrid: Ministerio de Defensa.

Littleton, J. (1966). The New Comparative Mythology. An Anthropological Assessment of the Theories of Georges Dumézil. Berkeley: The University of California Press.

Martínez, C. (2012). De Paraguay al piedemonte, de amigos a adversarios: Ñuflo de Chaves y los guaraníes en la conquista de Santa Cruz de la Sierra Santa Cruz la Vieja. En Combès, I. y Peña, P. (Eds.), Santa Cruz la Vieja (pp. 33-66). Santa Cruz de la Sierra: Fondo Editorial del Gobierno Autónomo.

Menget, P. (1985). Jalons pour une étude comparative. Journal de la Société des Américanistes, 71, 131-141.

Métraux, A. (1928). La Religion des Tupinambas et ses rapports avec celle des autres tribus Tupi-Guarani. París: ErnestLeroux.

Métraux, A. (1933). La guerra primitiva en el Chaco. La Prensa, 8 de enero de 1933, Sección Tercera, Buenos Aires.

Mitchell, P. (2015). Horse Nations: the worldwide impact of the horse on Indigenous societies post-1492. Oxford: Oxford University Press.

Momigliano, A. (1987). Georges Dumézil and the Trifunctional Approach to Roman Civilization.En Momigliano, A., Ottavo contributo alla storia degli studi classici e del mondo antico (pp. 135-159). Roma: Storia e Letteratura. 
Nordenskiöld, E. (2002 [1912]) La vida de los Indios. El Gran Chaco (Sudamérica). La Paz: APCOBPlural.

Oberg, K. (1949). The Terena and Caduveu of Southern Mato Grosso. Washington: Smithsonian Institution.

Otterbein, K. (1999). A History of Research on Warfare in Anthropology. American Anthropologist (n.s.), 101 (4), 794-805.

Otto, T., Thrase, H. y Vandkilde, H. (Eds.) (2006).Warfare and Society. Archaeological and Social Anthropological Perspectives. Aarhus: Aarhus University Press.

Rengger, J. R. (2010 [1835]). Viaje al Paraguay en los años 1818 a 1826. Asunción: Tiempo de Historia.

Richard, N. (Ed.) (2008). Mala guerra. Los indígenas en la guerra del Chaco (1932-1935). París -

Asunción: CoLibris - Museo del Barro - Servilibro.

Sahlins, M. (2000). Jungle Fever (review of 'Darkness in El Dorado' by Patrick Tierney). The Washington Post, Diciembre 10.

Saignes, T. (1985). La guerre contre l'histoire: les chiriguano du XVIe au XIXe siècle. Journal de la Société des Américanistes, 71, 175-190.

Sánchez Labrador, J. (1910 [c. 1770]). El Paraguay católico. Buenos Aires: Coni Hermanos.

Schindler, H. (1983). Die Reiterstämme des Gran Chaco. Berlín: Dietrich Reimer.

Steel, D. (1999). Trade Goods and Jívaro Warfare: The Shuar 1850-1957, and the Achuar, 1940-1978. Ethnohistory, 46 (4), 745-776.

Sterpin, A. (1993). La chasse aux scalps chez les Nivacle du Gran Chaco. Journal de la Société des Américanistes, 79, 33-66.

Susnik, B. (1971). El indio colonial del Paraguay, 3. El Chaqueño: Guaycurúes y Chanés-Arawak. Asunción: Museo Etnográfico Andrés Barbero.

Susnik, B. y Chase-Sardi, M. (1995). Los Indios del Paraguay. Madrid: Mapfre.

Taylor, A-C. (1985). L'art de la réduction: la guerre et les mécanismes de la différenciation tribale dans la culture jivaro. Journal de la Société des Américanistes, 71, 159-173.

Terray, E. (1989). Une Nouvelle anthropologie politique? L'Homme, 29 (110), 5-29.

Vernant, J-P. (1994). La guerra de las ciudades. En Vernant, J-P., Mito y sociedad en la Grecia antigua (pp. 22-45). Madrid: Siglo XXI.

Viveiros de Castro, E. (1992). From the Enemy's Point of View.Humanity and Divinity in an Amazonian Society. Chicago: University of Chicago Press.

Weber, M. (1993). Ensayos sobre metodología sociológica. Buenos Aires: Amorrortu.

\section{NOTES}

1. Agradezco a Nicolás Richard, Lorena Córdoba y particularmente a Kathleen Lowrey por haber contribuido de diversas formas con la realización de este trabajo.

2. Para balances críticos sobre el modelo duméziliano de las tres funciones, y en particular de la “segunda función” guerrera (Dumézil 1969), ver Littleton 1966; Momigliano 1987.

3. Entre las muchas lecturas críticas o apologéticas del legado clastreano pueden consultarse Saignes 1985; Terray 1989; Deleuze y Guattari 2002; Combès y Villar 2013; Campagno (Ed.) 2014. 
4. Entre otros, ver por ej. Menget 1985; Chaumeil 1985; Viveiros de Castro 1992; Descola 1993; Taylor 1995; Erikson 1986; Sterpin 1993, e incluso, en un contexto alejado de las tierras bajas sudamericanas, Vernant 1994. Una visión matizada de este tipo de enfoque es la de Goés Neves (2009).

5. Para más datos sobre la notable estratificación social de los caduveos, ver Sánchez Labrador 1910 [c. 1770]; Rengger 2010 [1835]; Oberg 1949; Susnik 1971; Cardoso de Oliveira 1976; Cordeu 2004. Para profundizar el tema del llamado "complejo ecuestre", ver Kersten 1968; Schindler 1983; Mitchell 2015.

6. Las repercusiones de las "guerras yanomamis", relativas tanto a las teorías de Chagnon como al legado de otros autores posteriores (J. Lizot, J. Neel, P. Tierney, etc.), son prácticamente inabarcables: ver por ej. Ferguson 1990, 2001; Heinen e Illius 1996; Sahlins 2000.

7. Como "esencialismo", "funcionalismo" es una de esas categorías que la corrección política actual impone conjugar como verbo irregular: yo estudio el sentido de determinada práctica para los actores, tú analizas la racionalidad de esa costumbre para una sociedad, él es funcionalista. Los funcionalistas son siempre los demás. ¿Pero cómo analizar la guerra sin preguntar "por qué" o "para qué"? ¿Dónde hay una interpretación de la guerra indígena que no sea teleológica en algún punto?

\section{AUTHOR}

\section{DIEGO VILLAR}

Consejo Nacional de Investigaciones Científicas y Técnicas, Argentina

Correo electrónico: villardieg@gmail.com 